\title{
Posizionamento ecoguidato di cateteri venosi centrali
}

\author{
Maurizio Gallieni
}

\section{U.O. di Nefrologia e Dialisi, Azienda Ospedaliera San Paolo, Polo Universitario, Milano}

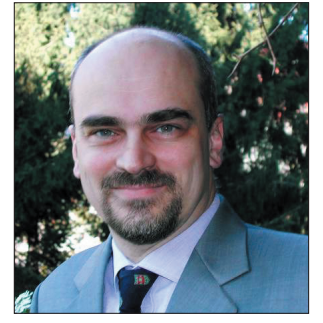

1 posiziona-
mento di un ca-
tetere venoso
centrale è una
procedura fonda-
mentale nella ge-
stione del paziente nefropatico (per l'esecuzione di metodiche che richiedono una circolazione extracorporea come emodialisi, emofiltrazione veno-venosa continua o CVVH, plasmaferesi), ma anche in altri ambiti clinici (misurazione della pressione venosa centrale, mancanza di accessi vascolari periferici, nutrizione parenterale, inserimento di pace-makers).

La vena giugulare interna è l'accesso venoso di prima scelta nel paziente dializzato

Le vene centrali utilizzate per le procedure elencate sono la vena succlavia, la vena giugulare interna e la vena femorale.

In ambito nefrologico è emersa di recente la convinzione che l'utilizzo della vena succlavia sia possibilmente da evitare per il verificarsi di ste- nosi della vena indotte dal decubito del catetere sulla parete venosa nel tratto curvilineo del vaso. Tali stenosi diventano clinicamente evidenti e importanti dopo il confezionamento chirurgico di un accesso vascolare all'arto superiore dove è presente la stenosi: si rilevano allora elevate pressioni venose durante il trattamento dialitico, edema dell'arto che può rendere difficoltosa la puntura dell'accesso vascolare e in casi estremi richiede la correzione della stenosi con angioplastica o addirittura la chiusura della fistola artero-venosa per emodialisi (1). Inoltre la presenza della stenosi preclude l'utilizzo dell'arto per la creazione di ulteriori accessi vascolari.

La vena femorale è di facile reperimento e l'inserimento del catetere presenta un rischio contenuto; è perciò largamente utilizzata nelle dialisi eseguite in urgenza, ma è teoricamente soggetta a un maggior rischio di complicanze infettive, legate alla presenza del catetere in una sede, la regione inguinale, facilmente inquinabile.

La vena giugulare interna rappresenta quindi il sito di prima scelta per il posizionamento di un catetere venoso per emodialisi. Non è stato definito se l'incidenza di trombosi sia inferiore a quella osservata nel cateterismo della vena succlavia. In effetti, a mano a mano che cresce l'esperienza con questo tipo di approccio è emerso che in diversi casi può svilupparsi trombosi, che resta clinicamente più silente in quanto non evidenziata (come invece succede per la vena succlavia) dalla creazione di una fistola artero-venosa. Un ulteriore vantaggio rispetto al posizionamento del catetere in vena succlavia è una maggior distanza dalla cupola pleurica e quindi una minor possibilità di pneumotorace.

Percentuali di successo e complicazioni della metodica di inserimento dei cateteri venosi centrali basata sui reperi anatomici esterni

La metodica tradizionale di posizionamento del catetere è quella basata sui reperi anatomici esterni. Il successo del posizionamento di un catetere nella vena succlavia o nella vena giugulare interna dipende dalle se- 
guenti circostanze: normale posizione, normale calibro e pervietà della vena. Le percentuali di successo del reperimento della vena con la metodica tradizionale sono variabili dal $67 \%$ al $96 \%$ (2-5). La variabilità di questi risultati dipende dall'esperienza dell'operatore e dalle circostanze in cui viene eseguita la procedura. Secondo Bo-Lin et al (2) la percentuale di successo è superiore nei casi di posizionamento del catetere in elezione rispetto alle procedure urgenti (che devono essere eseguite nell'arco di 1 ora) ed è marcatamente inferiore in caso di arresto cardiocircolatorio: su 460 casi le percentuali di successo sono state rispettivamente dell' $85 \%(120 / 142)$, del $76 \%(205 / 271)$ e del $62 \%(29 / 47)$.

Le complicazioni della procedura riportate in letteratura sono: puntura involontaria dell'arteria carotide nel 3$8 \%$ dei casi $(2,5)$, ematoma da puntura della carotide nell' $1 \%$ dei casi (3), pneumotorace nel $2-6 \%$ dei casi (2). Sebbene la puntura della carotide, se riconosciuta immediatamente, possa essere normalmente controllata con facilità per mezzo della compressione diretta del vaso, un eventuale inserimento di un catetere in un'arteria può determinare la formazione di un grave ematoma ed essere anche una complicazione letale $(6,7)$.
Confronto tra metodica basata sui reperi anatomici esterni e metodica ecoguidata

Due studi controllati eseguiti su di un ampio numero di pazienti hanno paragonato la metodica di incannulazione tradizionale con una metodica sotto guida ecografica $(4,5)$. I risultati di questi studi, con cui può essere confrontata la nostra casistica (8), sono riassunti in Tabella I. Questi studi, come pure la nostra esperienza, dimostrano che la puntura ecoguidata riduce il tempo richiesto per la procedura, riduce il numero di tentativi necessari a ottenere l'accesso venoso, aumenta la percentuale di successo finale alla totalità dei casi e minimizza le complicazioni come la puntura carotidea. Anche per la vena succlavia è descritto un miglioramento della performance dell'operatore che abbia a disposizione una guida ecografica in tempo reale. Thompson et al (9) hanno studiato 52 pazienti in cui sono stati posizionati cateteri venosi in vena succlavia da medici inesperti (che avevano eseguito precedentemente meno di 10 procedure). Sotto guida ecografica la percentuale di successo è stata del $92 \%$ (23/25 pazienti), mentre con la metodica tradizionale la procedura è stata di successo solo nel $44 \%$ dei casi $(12 / 27$ pazienti). Un altro studio ha utilizzato la metodica tradizionale con o senza precedente localizzazione ecografica della vena, eseguita da un diverso operatore e non nel momento della puntura (10). Le percentuali di insuccesso e di complicazioni erano sovrapponibili nei due gruppi di pazienti. La percentuale di insuccessi in questo studio $(12 \%)$ è molto elevata, e come sottolineato dagli stessi autori e da un editoriale di accompagnamento all'articolo (11), il fatto di non aver eseguito la procedura sotto guida ecografica diretta, "in tempo reale", ha probabilmente condizionato i risultati dello studio. Infatti numerosi altri studi hanno dimostrato, sia per la vena succlavia che per la vena giugulare interna, la superiorità della metodica eco-guidata $(4,5,9,12-19)$.

\section{Uno studio ecografico prelimina- re permette di definire l'anato- mia vascolare del paziente e di individuare il punto migliore di accesso}

La vena giugulare interna normalmente è localizzata in posizione laterale e lievemente più superficiale rispetto all'arteria carotide. La posizione di Trendelenburg e ancor più, nei pazienti collaboranti, la manovra di Valsalva aumentano il diametro della vena in modo rilevante. Infatti duran-

TABELLA I - RISULTATI $( \pm$ SD) DELLA TECNICA ECOGUIDATA DI INSERIMENTO DI CATETERI VENOSI CENTRALI IN VENA GIUGULARE INTERNA, PARAGONATI ALLA METODICA BASATA SUI REPERI ANATOMICI ESTERNI

\begin{tabular}{|c|c|c|c|c|c|}
\hline & \multirow{2}{*}{$\begin{array}{c}\text { Gallieni }(8) \\
\text { (pazienti nefrologici) } \\
\text { Eco } \\
(\mathbf{n}=\mathbf{2 1 0})\end{array}$} & \multicolumn{2}{|c|}{$\begin{array}{c}\text { Troianos et al (4) } \\
\text { (anestesia-rianimazione) }\end{array}$} & \multicolumn{2}{|c|}{$\begin{array}{l}\text { Denys et al (5) } \\
\text { (cardiologici) }\end{array}$} \\
\hline & & $\begin{array}{c}\text { Eco } \\
(\mathbf{n}=77)\end{array}$ & $\begin{array}{l}\text { Controlli } \\
(\mathrm{n}=83)\end{array}$ & $\begin{array}{c}\text { Eco } \\
(\mathbf{n}=928)\end{array}$ & $\begin{array}{l}\text { Controlli } \\
(\mathrm{n}=302)\end{array}$ \\
\hline Percentuale di successo & $\begin{array}{l}209 / 210 \\
(99.5 \%)\end{array}$ & $\begin{array}{c}77 / 77 \\
(100 \%)\end{array}$ & $\begin{array}{l}80 / 83 \\
(96 \%)\end{array}$ & $\begin{array}{l}928 / 928 \\
(100 \%)^{*}\end{array}$ & $\begin{array}{c}266 / 302 \\
(88 \%)\end{array}$ \\
\hline Successi al primo tentativo & $178(85 \%)$ & $56(73 \%)^{\S}$ & $45(54 \%)$ & $722(78 \%)^{*}$ & $116(37 \%)$ \\
\hline $\mathrm{N}$ di tentativi/paziente & $1.2 \pm 0.6$ & $1.4 \pm 0.7^{\S}$ & $2.8 \pm 3.0$ & $1.3 \pm 0.8 *$ & $2.5 \pm 2.7$ \\
\hline Punture arteriose & $6(2.9 \%)$ & $1(1.4 \%)$ & $7(8.4 \%)$ & $16(1.7 \%)^{*}$ & $25(8.3 \%)$ \\
\hline
\end{tabular}

$\S \mathrm{p}<0.05$ rispetto ai controlli

$* \mathrm{p}<0.001$ rispetto ai controlli 
te la manovra di Valsalva il diametro della vena giugulare interna può raddoppiare, mentre le dimensioni dell'arteria carotide restano sostanzialmente invariate. In letteratura sono descritte variazioni medie di diametro del $126 \%$ per la vena giugulare interna destra e del $70 \%$ per la sinistra rispetto al diametro a riposo (20). In uno studio ecografico eseguito su 200 pazienti (21) è stato rilevato che le vene giugulari interne sono normali per dimensioni e posizione nel $91.5 \%$ dei casi. Quindi nell' $8.5 \%$ dei casi la puntura della vena giugulare interna eseguita basandosi sui reperi anatomici esterni può essere un insuccesso (per la presenza di una trombosi venosa, di una vena molto piccola o con una posizione eccessivamente laterale) o determinare una puntura carotidea perché la vena è anteriore anziché laterale all'arteria. Questa percentuale corrisponde in effetti al numero medio di insuccessi riportati con la metodica tradizionale basata sui reperi anatomici esterni. Anche nei pazienti pediatrici è stata riportata un'anatomia venosa anomala nel $18 \%$ dei pazienti (22).

Lo svantaggio principale della metodica eco-assistita è rappresentato dal costo aggiuntivo dell'apparecchiatura. Tuttavia il significativo miglioramento dei risultati, con la maggiore sicurezza di poter posizionare il catetere anche nei casi più difficili senza dover chiedere aiuto all'anestesistarianimatore, il numero ridotto di complicazioni (e quindi di ospedalizzazione), la riduzione del tempo necessario alla procedura e la riduzione del numero di kit di inserzione utilizzati per paziente controbilanciano rapidamente il costo iniziale. Inoltre, sebbene non rientri nella valutazione dei costi economici, va ricordato che un elemento importante è il minor disagio del paziente legato alla riduzione significativa del numero di tentativi di puntura del vaso.

Anche l'aspetto medico-legale va tenuto in considerazione: l'operatore che deve rendere conto di una complicazione, che pur in una percentuale bassa è inevitabile in questo tipo di procedura, potrà sempre far presente che ha operato nelle migliori condi- zioni di sicurezza se ha utilizzato la metodica eco-guidata. Si raccomanda peraltro di far firmare al paziente un consenso informato alla procedura, tranne che nelle rare urgenze in cui ci sia pericolo di vita e in cui il paziente non è in grado di firmare.

\section{Tecnica di inserimento di catete- re in vena giugulare interna sot- to guida ecografica}

Prima di iniziare la procedura è senz'altro consigliabile studiare con l'apparecchio ecografico l'anatomia vascolare del paziente per selezionare il vaso e il punto più adatti di incannulazione. La vena giugulare può essere identificata per: 1) Posizione laterale e superficiale rispetto alla carotide; 2) Comprimibilità esterna rispetto alla carotide, il cui diametro non viene modificato dalla manovra e che presenta inoltre una pulsatilità; 3) Variazioni delle dimensioni con gli atti del respiro; una riduzione di diametro durante l'inspirazione indica inoltre con ragionevole certezza la pervietà delle vene anonima e cava superiore; 4) Aumento del diametro con la manovra di Valsalva.

$\mathrm{Nel}$ nostro Centro viene utilizzato un apparecchio ecografico portatile, (SITE-RITE II, Bard - Dymax, Pittsburgh, PA, USA; distribuito in Italia da Bellco SpA, Mirandola), di piccole dimensioni e alimentato da batterie ricaricabili. Lo strumento è quindi facilmente trasportabile e può essere utilizzato anche al letto del malato.

Per inserire un catetere venoso centrale è consigliabile utilizzare un letto senza testata per poter prendere posizione dietro la testa del paziente. Sia il paziente che l'operatore devono indossare mascherina e cappello, e va preparato accuratamente un campo sterile. Per garantire la sterilità del campo operatorio, si posiziona un apposito involucro in plastica trasparente al di sopra della sonda. La trasmissione degli ultrasuoni viene poi garantita dall'uso di gel sterile o di soluzione fisiologica applicata direttamente sulla cute del paziente. La sonda ecografica viene tenuta con la mano sinistra, mentre la mano destra manovra una siringa collegata all'ago già inserito nella guida. L'ago va fatto avanzare lentamente, a piccoli passi, tenendo gli occhi sul monitor dello strumento, che indicherà se la posizione del complesso sonda più ago è corretta. Una volta all'interno del vaso, la siringa tenuta in aspirazione si riempirà di sangue, il cui colore $\mathrm{e}$ pressione daranno già delle indicazioni sulla effettiva natura venosa. L'ago può a questo punto essere liberato dalla sonda e la procedura di inserimento del catetere prosegue secondo la nota tecnica di Seldinger. Dopo aver inserito la guida metallica nel vaso attraverso l'ago e aver rimosso l'ago dalla cute del paziente è possibile verificare se la guida sia effettivamente nella vena. La guida metallica è facilmente identificabile con una scansione ecografica, apparendo come un punto marcatamente iperecogeno all'interno del vaso o addossato alla sua parete.

In conclusione la disponibilità di una guida ecografica per ottenere l'accesso alla vena giugulare interna riduce in modo significativo le complicazioni e permette di raggiungere una percentuale di successi estremamente elevata con un minor numero di tentativi. La visualizzazione diretta della vena nel momento in cui si esegue la procedura permette di valutare con precisione le dimensioni e lo stato del vaso, aumentando il grado di sicurezza dell'operatore e il comfort del paziente. In un editoriale su questo argomento Scott (23) ha citato Erasmo da Rotterdam: "Nel modo dei ciechi l'orbo è re", sottolineando come la possibilità di visualizzare le strutture vascolari dia un vantaggio enorme all'operatore.

Senza dubbio la metodica ecoguidata è già ora consigliabile in tutti i Centri nei casi ad alto rischio (mancanza di reperi anatomici esterni, coagulopatie, precedenti cateterismi venosi), ma dovrebbe diventare in futuro l'approccio standard per l'inserimento dei cateteri venosi centrali per emodialisi. Dove già disponibile, come nel nostro Centro, la tecnica ecoguidata è già utilizzata in modo routinario in tutti i pazienti.

maurizio.gallieni@fastwebnet.it 


\section{BIBLIOGRAFIA}

1. Schwab SJ, Quarles LD, Middleton JP, Cohan RH, Saeed M, Dennis VW. Hemodialysis-as sociated subclavian vein stenosis. Kidney Int 1988; 33: 1156-9.

2. Bo-Lin GW, Anderson DJ, Anderson KC, McGoon MD. Percutaneous central venous catheterization performed by medical house officers: a prospective study. Cathet Cardiovasc Diagn 1982; 8: 23-9.

3. Goldfarb G, Lebrec D. Percutaneous cannulation of the internal jugular vein in patients with coagulopathies: An experience based on 1,000 attempts. Anesthesiology 1982; 56: 321-3.

4. Troianos CA, Jobes DR, Ellison N. Ultrasound-guided cannulation of the internal jugular vein. A prospective, randomized study. Anesth Analg 1991; 72: 823-6.

5. Denys BG, Uretsky BF, Reddy PS. Ultrasound assisted cannulation of the internal jugular vein. A prospective comparison to the external landmark guided technique. Circulation 1993; 87: 1557-62.

6. Jobes DR, Schwartz AJ, Greenhow DE, Stephenson LW, Ellison N. Safer jugular vein cannulation: recognition of arterial puncture and preferential use of the external jugular route. Anesthesiology 1983; 59: 353-5.

7. Morgan RNW, Morrell DF. Internal jugular catheterization: a review of a potentially lethal hazard. Anesthesia 1981; 36: 512-7.

8. Gallieni M. Central vein catheterization of dialysis patients with real time ultrasound guidance. The Journal of Vascular Access 2000; 1: $10-4$.

9. Thompson DR, Gualtieri E, Deppe S, Sipperly ME. Greater success in subclavian vein cannulation using ultrasound for inexperienced operators (abstr). Crit Care Med

10. 1994:22: A189 Mansfield PF, Hohn DC, Fornage BD, Gregurich MA, Ota DM. Complications and failures of subclavian vein catheterization. $\mathrm{N}$ Eng1 J Med 1994; 331: 1735-8.

11. Haire WD, Lieberman RP. Defining the risks of subclavian-vein catheterization. N Engl J Med 1994; 331: 1769-70.

12. Cavatorta F, Zollo A, Campisi $\mathrm{S}$, et al. Internal jugular vein catheterization under echographic guidance, Int J Artif Organs 1993; 16 : $820-2$.

13. Mallory DL, McGee WT, Shawker TH, et al. Ultrasound guidance improves the success rate of internal jugular vein cannulation. A prospective, randomized trial. Chest 1990; 98: 157-60.

14. Johnson R, O’Donnel J, Fielder K. Ultrasound guidance for cannulation of the internal jugular vein (IJV) in the critically ill. A randomized prospective study (abstr). Crit Care Med 1994; 22: A28.

15. Koski EM, Suhonen M, Mattila MA. Ultrasound-facilitated central venous cannulation. Crit Care Med 1992; 20: 424-6.

16. Alderson PJ, Burrows FA, Stemp LI, Holtby HM. Use of ultrasound to evaluate internal jugular vein antomy and to facilitate central venous cannulation in pediatric patients. Brit J Anaesth 1993; 70: 145-8.

17. Lameris JS, Post PJ, Zonderland HM, Gerristen PG, KappersKlunne MC, Schutte HE. Percutaneous placement of Hickman catheters: comparison of sonographically guided and blind techniques. AJR Am J Roentgenol 1990; 155: 1097-9.

18. Hull JE, Hunter CS, Luiken GA. The Groshong catheter: initial experience and early results of imaging-guided placement. Radiology 1992; 185: 803-7.

19. Gallieni M, Cozzolino M. Un- complicated central vein catheterization of high risk patients with real time ultrasound guidance. Int $\mathrm{J}$ Artif Org ans 1995; 18: 117-21.

Weissleder R, Elizondo G, Stark DD. Sonographic diagnosis of subclavian and internal jugular vein thrombosis. J Ultrasound Med 1987; 6: 577-87.

21. Denys BG, Uretsky BF. Anatomical variations of internal jugular vein location: impact on central venous access. Crit Care Med 1991; 19: 1516-9.

22. Alderson PJ, Burrows FA, Stemp LI, Holtby HM. Use of ultrasound to evaluate internal jugular vein anatomy and to facilitate central venous cannulation in paediatric patients. Br J Anaesth 1993; 70: $145-8$.

23. Armstrong PJ, Sutherland R, Scott DH. The effect of position and different manoeuvres on internal jugular vein diameter size. Acta Anaesthesiol Scand 1994; 38: 22931.

24. Scott DHT. "In the Country of the Blind, the One-Eyed Man is King," Eras mus (1466-1536). An Editorial. Brit J Anaesth 1999; 82: 820-1. 